\title{
Weekend Effect in Emergency Colon and Rectal Cancer Surgery: A Prospective Study Using Data From the Dutch ColoRectal Audit
}

\author{
Daniëlle D. Huijts, MSc ${ }^{\mathrm{a}}$; Julia T. van Groningen, $\mathrm{MD}^{\mathrm{b}}$; Onno R. Guicherit, $\mathrm{MD}, \mathrm{PhD}^{\mathrm{c}}$; \\ Jan Willem T. Dekker, MD, PhD'; Leti van Bodegom-Vos, PhD; ; Esther Bastiaannet, $\mathrm{PhD}^{\mathrm{e}}$; \\ Johannes A. Govaert, MD, PhD; ${ }^{\mathrm{e}}$ Michel W. Wouters, MD, PhD ${ }^{\mathrm{b}, \mathrm{f}}$; and Perla J. Marang-van de Mheen, $\mathrm{PhD}^{\mathrm{a}}$
}

\begin{abstract}
Background: It is unclear whether emergency weekend colon and rectal cancer surgery are associated with worse outcomes (ie, weekend effect) because previous studies mostly used administrative data, which may insufficiently adjust for case-mix. Materials and Methods: Prospectively collected data from the 2012-2015 Dutch ColoRectal Audit $(n=5,224)$ was used to examine differences in 30-day mortality and severe complication and failure-to-rescue rates for emergency weekend (Saturday and Sunday) versus Monday surgery, stratified for colon and rectal cancer. Analyses were adjusted for age, sex, body mass index, Charlson comorbidity index, American Society of Anesthesiologists classification score, tumor stage, presence of metastasis, preoperative complication, additional resection for metastasis or locally advanced tumor, location primary colon tumor, type of rectal surgery (lower anterior resection or abdominal perineal resection), and type of neoadjuvant therapy (short-course radiotherapy or chemoradiotherapy). Results: A total of 5,052 patients undergoing colon cancer surgery and 172 undergoing rectal cancer surgery were included. Patients undergoing colon or rectal cancer surgery during weekends had significantly more preoperative tumor complications compared with those undergoing surgery on a weekday. Additionally, differences in year of surgery and location of primary tumor were found for colon cancer surgery. Emergency colon cancer surgery during the weekend was associated with increased 30-day mortality (odds ratio [OR], 1.66; 95\% Cl, 1.10-2.50) and severe complications (OR, 1.29; 95\% Cl, 1.03-1.63) compared with surgery on Monday. Estimates for emergency weekend rectal cancer surgery were similar but not statistically significant, likely explained by small numbers. Conclusions: Weekend emergency colon cancer surgery was associated with higher mortality and severe complication rates. More research is needed to understand which factors explain and contribute to these differences.
\end{abstract}

J Natl Compr Canc Netw 2018;16(6):735-741 doi: 10.6004 /jnccn.2018.7016

\section{Background}

Colorectal cancer (CRC) is a frequently diagnosed cancer for which surgery has a crucial role in treatment. Unfortunately, surgery also carries considerable risks of postoperative complications and mortality.,2 There are indications that these risks might be higher in sur-

\footnotetext{
aDepartment of Biomedical Data Sciences, Medical Decision Making, Leiden University Medical Center, Leiden; 'butch Institute for Clinical Auditing, Leiden; 'Department of Surgery, University Cancer Center Leiden|The Hague, The Hague; ${ }^{d}$ Department of Surgery, Reinier de Graaf Group, Delft; 'Department of Surgery, Leiden University Medical Center, Leiden; and fDepartment of Surgical Oncology, Netherlands Cancer Institute-Antoni van Leeuwenhoek, Amsterdam, the Netherlands. Submitted December 14, 2017; accepted for publication February 20, 2018. The authors have disclosed that they have no financial interests, arrangements, affiliations, or commercial interests with the manufacturers of any products discussed in this article or their competitors.
}

geries performed during the weekend compared with weekdays: the so-called weekend effect. To date, a small number of studies have investigated the weekend effect in colorectal surgery. ${ }^{3-8}$ However, these studies grouped colon and rectal surgeries together ${ }^{3,4}$ or only examined

This study was funded by an unrestricted grant from the Dutch Cancer Society (UL 2015-7772). The Dutch Cancer Society did not influence the article in any way.

Author contributions: Study design: Guicherit, Marang-van de Mheen. Data analysis and interpretation: Huijts, van Groningen, Dekker, Marangvan de Mheen. Manuscript preparation: Huijts, Marang-van de Mheen. Critical revision: van Groningen, Guicherit, Dekker, van Bodegom-Vos, Bastiaannet, Govaert, Wouters, Marang-van de Mheen.

Correspondence: Perla J. Marang-van de Mheen, PhD, Department of Biomedical Data Sciences, Medical Decision Making, Leiden University Medical Center, Albinusdreef 2, 2333 ZA, Leiden, the Netherlands. Email: p.j.marang@lumc.n 
Huijts et al

colon surgery, ${ }^{5,6}$ whereas patient characteristics, complication risks, and clinical outcomes are clearly different for colon and rectal cancers. ${ }^{9}$

Another important limitation of most previous studies on the weekend effect, regardless of the diagnosis, is the use of administrative data. Administrative data can reflect inaccurate coding, ${ }^{10}$ but also often lack necessary information to adjust for the different case-mix using clinical variables. ${ }^{11,12}$ As a result, whether the weekend effect is merely a product of the selection of worst cases presenting during the weekend or is caused by other factors related to the quality of care delivered on weekends remains unclear. However, when restricting to emergency cases, such selection is less likely to occur on weekends and not (during out-of-office hours) on weekdays. Due to these matters, there is an increasing demand for population-based data from clinical audits because these may provide the necessary clinical data for case-mix adjustment. The Dutch ColoRectal Audit (DCRA), previously known as the Dutch Surgical Colorectal Audit, is a nationwide clinical audit in the Netherlands with the objective to measure and improve quality of CRC surgery. It registers detailed clinical data to enable case-mix adjustment to make valid comparisons of outcomes of different providers. ${ }^{13}$

The aim of the current study, therefore, was to investigate whether there is a weekend effect after emergency colon and rectal cancer surgery using data from the DCRA.

\section{Materials and Methods}

\section{Study Design and Setting}

Data were prospectively collected from the DCRA, a nationwide quality improvement audit initiated in 2009. All Dutch hospitals $(n=88)$ performing CRC surgery participate in the DCRA. The DCRA is a disease-specific audit and contains a wide range of variables with specific information regarding patient and tumor characteristics, diagnostics, treatment, complications, and mortality. A more detailed description of the DCRA is published elsewhere. ${ }^{13,14}$

\section{Study Population}

All nonelective patients $(n=5,246)$ registered in the DCRA for surgical resection of primary CRC in the period from 2012 until 2015 were included. Patients with synchronous tumors, treated with the "wait-and-see" approach, who underwent procedures solely performed through transanal endoscopic microsurgery, and without information on date of surgery, mortality, or location of the primary tumor were excluded $(n=22)$. Elective resections were excluded because weekend elective surgery is not common practice in the Netherlands, and therefore any surgeries performed during the weekend were likely to be a specific selection of patients giving rise to treatment by indication bias. The study protocol was presented to the Medical Ethical Committee of the Leiden University Medical Center, which declared that no ethical approval was necessary for this type of study under Dutch law and waived the need for informed written consent from patients (P16.243).

\section{Data and Definitions}

Previous studies varied regarding whether day of admission or day of surgery was used to define the weekend; we used day of surgery to define the weekend (Saturday and Sunday). A more precise period (eg, including evening or nights) could not be given because no specific information on time of surgery was available.

Outcome measures were mortality, severe complication, and failure to rescue (FTR). Mortality was defined as postoperative death in the hospital during the same admission or within 30 days after resection. Severe complication was defined as any postoperative complication leading to a prolonged hospital stay of $>14$ days, a reintervention, or mortality. FTRs were defined as patients with a severe complication who died within 30 days after resection or during the same hospital admission. These definitions have also been used in previous DCRA studies. ${ }^{14-17}$

Case-mix variables used to adjust outcomes for both colon and rectal cancer surgery were age, sex, body mass index, Charlson comorbidity index, American Society of Anesthesiologists classification score, presence of metastasis, preoperative complications, and additional resection for metastasis or locally advanced tumor. Specific for colon cancer, the pathologic tumor stage and location of the primary tumor were added to the case-mix variables, and specific for rectal cancer, the clinical tumor stage, type of surgery (low anterior resection or abdominal perineal resection), and neoadjuvant therapy (shortcourse radiotherapy or chemoradiotherapy) were added. These case-mix variables have been previous- 
ly identified as relevant for patient outcome and hospital comparisons, and are thus part of the standard DCRA case-mix models. ${ }^{14}$

\section{Statistical Analysis}

First, patient characteristics were compared between weekend and weekday surgery, separately for colon and rectal cancer, using chi-square tests. Subsequently, logistic regression was used to examine whether unadjusted and adjusted outcome rates differed between weekend and weekday surgery. Monday was used as reference category. Analyses were adjusted for the case-mix variables. Goodness of fit of the adjusted models was assessed using C-statistics by calculating the area under the receiver operating characteristics curve. Results were considered statistically significant at $P<.05$. Statistical analyses were performed using SPSS version 23 (IBM Corporation).

\section{Sensitivity Analysis}

Sensitivity analyses were performed with all weekdays combined. Furthermore, because hospital care during Dutch national holidays is likely similar to weekend care, dates listed as national holidays for hospitals $(n=10)$ were coded as weekend. Subsequently, primary analyses were repeated.

\section{Results}

\section{Patient Characteristics}

A total of 5,224 patients receiving emergency care were included in the analyses, with 5,052 (96.7\%) undergoing colon cancer surgery and 172 (3.3\%) undergoing rectal cancer surgery. Among emergency colon cancer surgery, 4,244 $(84.0 \%)$ were performed during a weekday and 808 (16.0\%) during the weekend. Patients undergoing weekend emergency colon cancer surgery more often had preoperative tumor complications than weekday surgery patients (Table 1). Furthermore, the location of the primary tumor and year of surgery significantly differed, with relatively more weekend surgeries performed in recent years. Among emergency rectal cancer surgeries, 150 $(87.2 \%)$ were performed during a weekday and 22 $(12.8 \%)$ during the weekend. Preoperative tumor complications more often occurred in patients undergoing weekend emergency rectal cancer surgery (Table 1).

\section{Outcomes for Colon and Rectal Cancer Surgery}

In emergency colon cancer surgery (Table 2), the highest mortality, severe complication, and FTR rates were found for weekend surgeries $(9.9 \%, 31.8 \%$, and $31.1 \%$, respectively). After adjustment for casemix, weekend surgeries had $66 \%$ higher mortality and 29\% higher severe complication rates compared with Monday surgeries (Table 2). Estimates for FTR were in the same direction but not statistically significant, which is likely due to the smaller sample size as only complicated cases are included.

In emergency rectal cancer surgery, the highest mortality, severe complication, and FTR rates were found for weekend surgeries $(18.2 \%, 45.5 \%$, and $40.0 \%$, respectively). However, no significant differences were found for weekend surgeries after adjustment for case-mix, even though estimates went in the same direction as for colon cancer surgery, likely caused by the small numbers (Table 2).

\section{Sensitivity Analysis}

Combining all weekdays showed similar results (data not shown). Slight differences in the odds ratios (ORs) were observed, but the direction of the association remained the same. Coding surgeries performed on a Dutch national holiday as a weekend surgery $(n=76)$ also did not influence the results (data not shown).

As a post hoc analysis, we explored whether ostomies or anastomoses were more often performed during weekend or weekday surgery, because this may indicate either a different patient population not accounted for by case-mix correction and/or a difference in experience of the surgeon performing the surgery, and thereby suggest possible explanatory or mediating factors. For emergency colon cancer surgery, more ostomies were created on the weekend than on weekdays $(43.6 \%$ vs $38.8 \% ; P=.018)$. During weekdays, the percentage of anastomoses was higher compared with the weekend $(55.6 \%$ vs $50.1 \% ; P=.018)$. Including this as an additional variable (ostomy, anastomosis, or both) in the multivariate analyses, with having both an ostomy and anastomosis as the reference category, resulted in the FTR rate being significantly higher after emergency weekend colon cancer surgeries (OR, 1.66; 95\% CI, 1.04-2.66). 
Huijts et al

\begin{tabular}{|c|c|c|c|c|c|c|c|c|c|c|}
\hline & \multicolumn{5}{|c|}{ Colon } & \multicolumn{5}{|c|}{ Rectal } \\
\hline & \multicolumn{2}{|c|}{$\begin{array}{l}\text { Weekdays } \\
(\mathrm{N}=4,244)\end{array}$} & \multicolumn{2}{|c|}{$\begin{array}{l}\text { Weekend } \\
(\mathrm{N}=808)\end{array}$} & \multirow{2}{*}{$\frac{\text { Sign }}{P \text { Value }}$} & \multicolumn{2}{|c|}{$\begin{array}{l}\text { Weekdays } \\
(\mathrm{N}=150)\end{array}$} & \multicolumn{2}{|c|}{$\begin{array}{l}\text { Weekend } \\
(\mathrm{N}=22)\end{array}$} & \multirow{2}{*}{$\frac{\text { Sign }}{P \text { Value }}$} \\
\hline & $n$ & $\%$ & $n$ & $\%$ & & $n$ & $\%$ & $n$ & $\%$ & \\
\hline \multicolumn{11}{|l|}{ Sex } \\
\hline Male & 2,141 & $50.4 \%$ & 419 & $51.9 \%$ & NS & 91 & $61.1 \%$ & 14 & $66.7 \%$ & NS \\
\hline Female & 2,103 & $49.6 \%$ & 388 & $48.1 \%$ & & 58 & $38.9 \%$ & 7 & $33.3 \%$ & \\
\hline \multicolumn{11}{|l|}{ Age, y } \\
\hline$\leq 60$ & 759 & $17.9 \%$ & 143 & $17.7 \%$ & NS & 39 & $26.0 \%$ & 4 & $18.2 \%$ & NS \\
\hline $61-70$ & 1,253 & $29.5 \%$ & 214 & $26.5 \%$ & & 28 & $18.7 \%$ & 4 & $18.2 \%$ & \\
\hline $71-80$ & 1,293 & $30.5 \%$ & 254 & $31.4 \%$ & & 46 & $30.7 \%$ & 9 & $40.9 \%$ & \\
\hline$\geq 81$ & 938 & $22.1 \%$ & 197 & $24.4 \%$ & & 37 & $24.7 \%$ & 5 & $22.7 \%$ & \\
\hline \multicolumn{11}{|l|}{$\mathrm{BMI}, \mathrm{kg} / \mathrm{m}^{2}$} \\
\hline$<18.5$ & 122 & $3.2 \%$ & 35 & $5.0 \%$ & NS & 4 & $3.0 \%$ & 2 & $11.1 \%$ & NS \\
\hline $18.5-24.9$ & 1,872 & $49.2 \%$ & 337 & $48.3 \%$ & & 66 & $49.3 \%$ & 9 & $50.0 \%$ & \\
\hline $25.0-29.9$ & 1,303 & $34.3 \%$ & 246 & $35.2 \%$ & & 49 & $36.6 \%$ & 6 & $33.3 \%$ & \\
\hline$\geq 30.0$ & 505 & $13.3 \%$ & 80 & $11.5 \%$ & & 15 & $11.2 \%$ & 1 & $5.6 \%$ & \\
\hline \multicolumn{11}{|c|}{ Charlson comorbidity index } \\
\hline 0 & 2,188 & $51.6 \%$ & 424 & $52.5 \%$ & NS & 59 & $39.3 \%$ & 12 & $54.5 \%$ & NS \\
\hline 1 & 973 & $22.9 \%$ & 184 & $22.8 \%$ & & 45 & $30.0 \%$ & 5 & $22.7 \%$ & \\
\hline$\geq 2$ & 1,083 & $25.5 \%$ & 200 & $24.8 \%$ & & 46 & $30.7 \%$ & 5 & $22.7 \%$ & \\
\hline ASA score & & & & & & & & & & \\
\hline $1-I I$ & 2,657 & $62.7 \%$ & 506 & $62.6 \%$ & NS & 82 & $55.0 \%$ & 12 & $54.5 \%$ & NS \\
\hline III & 1,358 & $32.1 \%$ & 251 & $31.1 \%$ & & 54 & $36.2 \%$ & 9 & $40.9 \%$ & \\
\hline IV-V & 222 & $5.2 \%$ & 51 & $6.3 \%$ & & 13 & $8.7 \%$ & 1 & $4.5 \%$ & \\
\hline Tumor stage & & & & & & & & & & \\
\hline $\mathrm{X}^{\mathrm{b}}$ & 20 & $0.5 \%$ & 4 & $0.5 \%$ & NS & 33 & $22.0 \%$ & 9 & $40.9 \%$ & NS \\
\hline $0-I^{b}$ & 36 & $0.8 \%$ & 8 & $1.0 \%$ & & 4 & $2.7 \%$ & 0 & $0 \%$ & \\
\hline II & 176 & $4.1 \%$ & 38 & $4.7 \%$ & & 16 & $10.7 \%$ & 1 & $4.5 \%$ & \\
\hline III & 2,607 & $61.4 \%$ & 485 & $60.0 \%$ & & 69 & $46.0 \%$ & 10 & $45.5 \%$ & \\
\hline IV & 1,405 & $33.1 \%$ & 273 & $33.8 \%$ & & 28 & $18.7 \%$ & 2 & $9.1 \%$ & \\
\hline Metastasis & & & & & & & & & & \\
\hline No & 3,186 & $75.1 \%$ & 623 & $77.1 \%$ & NS & 107 & $71.3 \%$ & 16 & $72.7 \%$ & NS \\
\hline Yes & 1,058 & $24.9 \%$ & 185 & $22.9 \%$ & & 43 & $28.7 \%$ & 6 & $27.3 \%$ & \\
\hline Preoperative tumor con & ions & & & & & & & & & \\
\hline No & 398 & $9.4 \%$ & 46 & $5.7 \%$ & .001 & 36 & $24.0 \%$ & 0 & $0 \%$ & .010 \\
\hline Yes & 3,846 & $90.6 \%$ & 762 & $94.3 \%$ & & 114 & $76.0 \%$ & 22 & $100 \%$ & \\
\hline Additional resection fo & stasis & & & & & & & & & \\
\hline No & 4,056 & $95.6 \%$ & 776 & $96.0 \%$ & NS & 146 & $97.3 \%$ & 21 & $95.5 \%$ & NS \\
\hline Yes & 188 & $4.4 \%$ & 32 & $4.0 \%$ & & 4 & $2.7 \%$ & 1 & $4.5 \%$ & \\
\hline Additional resection fo & y advan & umor & & & & & & & & \\
\hline No & 3,613 & $85.1 \%$ & 709 & $87.7 \%$ & NS & 133 & $88.7 \%$ & 19 & $86.4 \%$ & NS \\
\hline Yes & 631 & $14.9 \%$ & 99 & $12.3 \%$ & & 17 & $11.3 \%$ & 3 & $13.6 \%$ & \\
\hline Location primary tumo & & & & & & & & & & \\
\hline Cecum & 719 & $16.9 \%$ & 147 & $18.2 \%$ & .038 & - & - & - & - & - \\
\hline Appendix & 37 & $0.9 \%$ & 9 & $1.1 \%$ & & - & - & - & - & \\
\hline Ascending colon & 595 & $14.0 \%$ & 80 & $9.9 \%$ & & - & - & - & - & \\
\hline Hepatic flexure & 253 & $6.0 \%$ & 58 & $7.2 \%$ & & - & - & - & - & \\
\hline Transverse colon & 381 & $9.0 \%$ & 89 & $11.0 \%$ & & - & - & - & - & \\
\hline Splenic flexure & 264 & $6.2 \%$ & 45 & $5.6 \%$ & & - & - & - & - & \\
\hline Descending colon & 399 & $9.4 \%$ & 78 & $9.7 \%$ & & - & - & - & - & \\
\hline Sigmoid colon & 1,596 & $37.6 \%$ & 302 & $37.4 \%$ & & - & - & - & - & \\
\hline Neoadjuvant therapy & & & & & & & & & & \\
\hline No & - & - & - & - & - & 104 & $69.3 \%$ & 19 & $86.4 \%$ & NS \\
\hline $\begin{array}{l}\text { Short-course } \\
\text { radiotherapy }\end{array}$ & - & - & - & - & & 30 & $20.0 \%$ & 0 & $0 \%$ & \\
\hline Chemoradiotherapy & - & - & - & - & & 16 & $10.7 \%$ & 3 & $13.6 \%$ & \\
\hline Procedure rectal & & & & & & & & & & \\
\hline LAR & - & - & - & - & - & 116 & $77.3 \%$ & 1 & $4.5 \%$ & NS \\
\hline APR & - & - & - & - & & 25 & $16.7 \%$ & 20 & $90.9 \%$ & \\
\hline Other & - & - & - & - & & 9 & $6.0 \%$ & 1 & $4.5 \%$ & \\
\hline Year of surgery & & & & & & & & & & \\
\hline 2012 & 1,171 & $27.6 \%$ & 190 & $23.5 \%$ & .033 & 47 & $31.3 \%$ & 6 & $27.3 \%$ & NS \\
\hline 2013 & 1,091 & $25.7 \%$ & 199 & $24.6 \%$ & & 30 & $20.0 \%$ & 6 & $27.3 \%$ & \\
\hline 2014 & 1,036 & $24.4 \%$ & 212 & $26.2 \%$ & & 44 & $29.3 \%$ & 7 & $31.8 \%$ & \\
\hline 2015 & 946 & $22.3 \%$ & 207 & $25.6 \%$ & & 29 & $19.3 \%$ & 3 & $13.6 \%$ & \\
\hline
\end{tabular}

Abbreviations: APR, abdominal perineal resection; ASA, American Society of Anesthesiologists; BMI, body mass index; LAR, low anterior resection; NS, not significant. aStatistically significant $P$ values are shown in bold.

'The pathologic tumor stage is used for colon and the clinical tumor stage is used for rectal. The first 2 stages for clinical tumor stage are stage 0 and $I$. 
Weekend Effect in Colon and Rectal Cancer Surgery

\section{Discussion}

The present study has shown higher mortality and severe complication rates after emergency colon cancer surgery performed during the weekend. Estimates for FTR and emergency rectum cancer surgery were in the same direction but not statistically significant, likely explained by the small sample size. By separating colon and rectal cancer, we believe that our results provide a more complete picture of the occurrence of a weekend effect in colorectal surgery and a better starting point for further research into explanatory factors and quality improvement initiatives.

Given the worse outcomes seen after emergency colon cancer surgery during weekends, it is important to better understand the complex mechanism that may result in the observed weekend effect. Because the weekend effect also remained after casemix adjustment using clinical variables, we eliminated the possibility that the effect might be caused by a case-mix selection, making differences in organization of perioperative care during weekends seem more likely. In an attempt to gain more insight into this mechanism, we explored whether having an anastomosis or an ostomy might be an explanatory or mediating factor, indicating either a specific patient group not accounted for in case-mix adjustment and/ or a less experienced surgical team. It can be argued that ostomies are less risky to perform and therefore more preferred among less specialized surgeons who might be on call on some weekends. However, the decision-making around ostomies and anastomoses is influenced by many other factors, with the condition of the patient being the most important, which might explain the increased FTR rate when this factor was included in the analysis. Therefore, it seems far more likely that this effect is the result of a complex combination of surgeon specialization and patient condition, requiring more research into the mechanism behind the observed weekend effect.

Future studies should shift the focus toward possible explanatory factors, such as organization of care during the weekends compared with weekdaysnot only whether the perioperative period is in the

Table 2. Outcomes for Emergency Colon and Rectal Cancer Surgery

\begin{tabular}{|c|c|c|c|c|c|c|c|}
\hline & Monday & Tuesday & Wednesday & Thursday & Friday & Weekend $^{\mathrm{a}}$ & C-Statistic \\
\hline \multicolumn{8}{|l|}{ Colon } \\
\hline Number of procedures & 723 & 792 & 863 & 894 & 972 & 808 & \\
\hline \multicolumn{8}{|l|}{ 30-day mortality } \\
\hline Mortality rate, $\mathrm{n}(\%)$ & $46(6.4)$ & $62(7.8)$ & $62(7.2)$ & $56(6.3)$ & $73(7.5)$ & $80(9.9)$ & \\
\hline Unadjusted OR $(95 \% \mathrm{Cl})$ & 1 & $1.25(0.84-1.86)$ & $1.14(0.77-1.69)$ & $0.98(0.66-1.47)$ & $1.20(0.82-1.75)$ & $1.62(1.11-2.36)$ & \\
\hline Adjusted OR $(95 \% \mathrm{Cl})$ & 1 & $1.29(0.84-1.97)$ & $1.17(0.76-1.79)$ & $0.95(0.61-1.47)$ & $1.23(0.81-1.86)$ & $1.66(1.10-2.50)$ & 0.792 \\
\hline \multicolumn{8}{|l|}{ Severe complication } \\
\hline Severe complication rate, $\mathrm{n}(\%)$ & $188(26.0)$ & $234(29.5)$ & $237(27.5)$ & $229(25.6)$ & $269(27.7)$ & $257(31.8)$ & \\
\hline Unadjusted OR $(95 \% \mathrm{Cl})$ & 1 & $1.19(0.95-1.50)$ & $1.08(0.86-1.35)$ & $0.98(0.78-1.23)$ & $1.09(0.88-1.35)$ & $1.33(1.06-1.66)$ & \\
\hline Adjusted OR $(95 \% \mathrm{Cl})$ & 1 & $1.19(0.94-1.50)$ & $1.06(0.84-1.33)$ & $0.96(0.76-1.21)$ & $1.08(0.86-1.35)$ & $1.29(1.03-1.63)$ & 0.659 \\
\hline \multicolumn{8}{|l|}{ Failure to rescue ${ }^{b}$} \\
\hline Failure to rescue rate, $\mathrm{n}(\%)$ & $46(24.5)$ & $62(26.5)$ & $62(26.2)$ & $56(24.5)$ & $73(27.1)$ & $80(31.1)$ & \\
\hline Unadjusted OR (95\% Cl) & 1 & $1.11(0.72-1.73)$ & $1.09(0.70-1.70)$ & $1.00(0.64-1.57)$ & $1.15(0.75-1.76)$ & $1.40(0.91-2.13)$ & \\
\hline Adjusted OR $(95 \% \mathrm{Cl})$ & 1 & $1.20(0.74-1.94)$ & $1.15(0.71-1.85)$ & $1.09(0.67-1.77)$ & $1.36(0.86-2.16)$ & $1.55(0.98-2.45)$ & 0.731 \\
\hline \multicolumn{8}{|l|}{ Rectal } \\
\hline Number of procedures & 24 & 34 & 37 & 26 & 29 & 22 & \\
\hline \multicolumn{8}{|l|}{ 30-day mortality } \\
\hline Mortality rate, n (\%) & $1(4.2)$ & $5(14.7)$ & $2(5.4)$ & $2(7.7)$ & $2(6.9)$ & $4(18.2)$ & \\
\hline Unadjusted OR (95\% Cl) & 1 & $3.97(0.43-36.35)$ & $1.31(0.11-15.34)$ & $1.92(0.16-22.61)$ & $1.70(0.14-20.02)$ & $5.11(0.52-49.79)$ & \\
\hline Adjusted OR $(95 \% \mathrm{Cl})$ & 1 & $5.73(0.53-62.56)$ & $1.23(0.09-16.62)$ & $0.91(0.05-17.28)$ & $0.84(0.05-15.60)$ & $6.33(0.56-71.41)$ & 0.812 \\
\hline \multicolumn{8}{|l|}{ Severe complication } \\
\hline Severe complication rate, $\mathrm{n}(\%)$ & $6(25.0)$ & $15(44.1)$ & $10(27.0)$ & $9(34.6)$ & $10(34.5)$ & $10(45.5)$ & \\
\hline Unadjusted OR $(95 \% \mathrm{Cl})$ & 1 & $2.37(0.75-7.45)$ & $1.11(0.34-3.60)$ & $1.59(0.47-5.42)$ & $1.58(0.48-5.24)$ & $2.50(0.72-8.71)$ & \\
\hline Adjusted OR $(95 \% \mathrm{Cl})$ & 1 & $2.26(0.69-7.44)$ & $1.06(0.31-3.59)$ & $1.08(0.29-4.04)$ & $1.22(0.35-4.26)$ & $1.76(0.47-6.55)$ & 0.642 \\
\hline \multicolumn{8}{|l|}{ Failure to rescue ${ }^{b}$} \\
\hline Failure to rescue rate, $\mathrm{n}(\%)$ & $1(16.7)$ & $5(33.3)$ & $2(20.0)$ & $2(22.2)$ & $2(20.0)$ & $4(40.0)$ & \\
\hline Unadjusted OR (95\% Cl) & 1 & $2.50(0.23-27.57)$ & $1.25(0.09-17.65)$ & $1.43(0.10-20.44)$ & $1.25(0.09-17.65)$ & $3.33(0.28-40.29)$ & \\
\hline Adjusted OR (95\% CI) & 1 & $8.12(0.26-251.72)$ & $1.74(0.06-49.48)$ & $0.75(0.02-32.03)$ & $1.86(0.04-89.06)$ & $12.10(0.38-387.90)$ & 0.788 \\
\hline
\end{tabular}

Abbreviation: $O R$, odds ratio.

asignificant values of $P<.05$ are shown bold.

${ }^{b}$ Failure to rescue rates and OR are based on the rates of severe complication. 
Huijts et al

weekend but also whether care given in the postoperative period is during the weekend. Factors suggested to explain the weekend effect are less (experienced) hospital staff and resources available during the weekends. ${ }^{18}$ This would suggest a broader focus, because it should also include delay in diagnosing complications and performing reinterventions, rather than only focusing on the initial operation being performed in the weekend. For instance, an anastomotic leakage, a common serious complication in colorectal surgery, is typically diagnosed between 5 and 8 days after surgery, ${ }^{19}$ so when this occurs during a weekend, it may result in higher FTR rates if not detected early enough, even though the primary surgery was performed on Monday or Tuesday. This will only be detected when looking specifically at delay during weekends in diagnosing complications and/ or performing reinterventions during weekends. One study, with a relatively small study population, observed intervening weekends to be a risk factor for delay in diagnosing anastomotic leakage and, subsequently, relaparotomy. ${ }^{20}$ In addition, a study into hospital characteristics and the effect on FTR following major surgery suggested that microsystem characteristics are likely to be more important in managing postoperative complications, because only part of the variability between low- and high-mortality hospitals could be explained by macrosystem factors such as hospital size. ${ }^{21}$ Therefore, it might be far more complicated than simply having specialized surgeons on call 24 hours a day or more beds available, but rather could be an interplay between availability of diagnostics, sufficient and experienced staff at the wards with the ability to recognize early signs of complications, and quick surgical interventions by specialized surgeons if needed.

This study has some limitations, one of which is that no information was available regarding outof-hospital deaths occurring $>30$ days after surgery.
However, for this to influence our results, the out-ofhospital deaths should have followed a different pattern than the included 30-day mortality, which does not seem likely. Another limitation may be our lack of information on day of admission, making it possible that weekend surgeries are represented by the worst cases and that other slightly less emergent surgeries are postponed to Monday. This might suggest an overestimation of the weekend effect we found. Use of the DCRA in the current study could be considered a strength, particularly because of the available clinical variables in contrast to previous studies that mostly used administrative data. Previous studies often report that they were unable to fully adjust for the different patient characteristics because of the lack of clinical data indicating a different casemix in weekends. ${ }^{18}$ Our results still show differences after adjusting for a broad scale of these clinical casemix variables, indicating that the weekend effect found in this study may possibly be due to a variation in care instead of a selection effect.

\section{Conclusions}

The current study demonstrated worse patient outcomes after emergency colon cancer surgery performed on weekends, reflecting a weekend effect. Future studies should examine explanatory factors, including differences in quality of care during weekends compared with weekdays in terms of diagnostic availability, surgical experience, and postoperative care that may affect the detection of early signs of complications.

\section{Acknowledgments}

The authors would like to thank all surgeons, registrars, physician assistants, and administrative nurses who registered the patients in the DCRA, as well as the DCRA group.

\section{References}

1. Ingraham AM, Cohen ME, Bilimoria KY, et al. Comparison of 30-day outcomes after emergency general surgery procedures: potential for targeted improvement. Surgery 2010;148:217-238.

2. Paun BC, Cassie S, MacLean AR, et al. Postoperative complications following surgery for rectal cancer. Ann Surg 2010;251:807-818.

3. Glance LG, Osler T, Li Y, et al. Outcomes are worse in US patients undergoing surgery on weekends compared with weekdays. Med Care 2016;54:608-615.

4. Zhou Y, Li W, Herath C, et al. Off-hour admission and mortality risk for 28 specific diseases: a systematic review and meta-analysis of 251 cohorts. J Am Heart Assoc 2016;5:e003102.

5. Aylin P, Yunus A, Bottle A, et al. Weekend mortality for emergency admissions. A large, multicentre study. Qual Saf Health Care 2010;19:213217.

6. Cram P, Hillis SL, Barnett M, et al. Effects of weekend admission and hospital teaching status on in-hospital mortality. Am J Med 2004;117:151-157.

7. Bell CM, Redelmeier DA. Mortality among patients admitted to hospitals on weekends as compared with weekdays. N Engl J Med 2001;345:663-668.

8. Hoehn RS, Go DE, Dhar VK, et al. Understanding the "weekend effect" for emergency general surgery. J Gastrointest Surg 2018;22:321-328.

9. van der Sijp MP, Bastiaannet E, Mesker WE, et al. Differences between colon and rectal cancer in complications, short-term survival and recurrences. Int J Colorectal Dis 2016;31:1683-1691. 
Weekend Effect in Colon and Rectal Cancer Surgery

10. Li L, Rothwell PM. Biases in detection of apparent "weekend effect" on outcome with administrative coding data: population based study of stroke. BMJ 2016;353:i2648.

11. Lilford RJ, Chen YF. The ubiquitous weekend effect: moving past proving it exists to clarifying what causes it. BMJ Qual Saf 2015;24:480-482.

12. Concha OP, Gallego B, Hillman K, et al. Do variations in hospital mortality patterns after weekend admission reflect reduced quality of care or different patient cohorts? A population-based study. BMJ Qual Saf 2014;23:215-222.

13. van Leersum NJ, Snijders HS, Henneman D, et al. The Dutch Surgical Colorectal Audit. Eur J Surg Oncol 2013;39:1063-1070.

14. Kolfschoten NE, Marang van de Mheen PJ, Gooiker GA, et al. Variation in case-mix between hospitals treating colorectal cancer patients in the Netherlands. Eur J Surg Oncol 2011;37:956-963.

15. Henneman D, Snijders HS, Fiocco M, et al. Hospital variation in failure to rescue after colorectal cancer surgery: results of the Dutch Surgical Colorectal Audit. Ann Surg Oncol 2013;20:2117-2123.
16. Henneman D, Ten Berge MG, Snijders HS, et al. Safety of elective colorectal cancer surgery: non-surgical complications and colectomies are targets for quality improvement. J Surg Oncol 2014;109:567-573.

17. Henneman $D$, van Leersum NJ, Ten Berge M, et al. Failure-to-rescue after colorectal cancer surgery and the association with three structural hospital factors. Ann Surg Oncol 2013;20:3370-3376.

18. Bray BD, Steventon A. What have we learnt after 15 years of research into the 'weekend effect'? BMJ Qual Saf 2017;26:607-610.

19. Daams F, Luyer M, Lange JF. Colorectal anastomotic leakage: aspects of prevention, detection and treatment. World J Gastroenterol 2013;19:2293-2297.

20. Doeksen A, Tanis PJ, Vrouenraets BC, et al. Factors determining delay in relaparotomy for anastomotic leakage after colorectal resection. World J Gastroenterol 2007;13:3721-3725.

21. Sheetz KH, Dimick JB, Ghaferi AA. Impact of hospital characteristics on failure to rescue following major surgery. Ann Surg 2016;263:692-697.

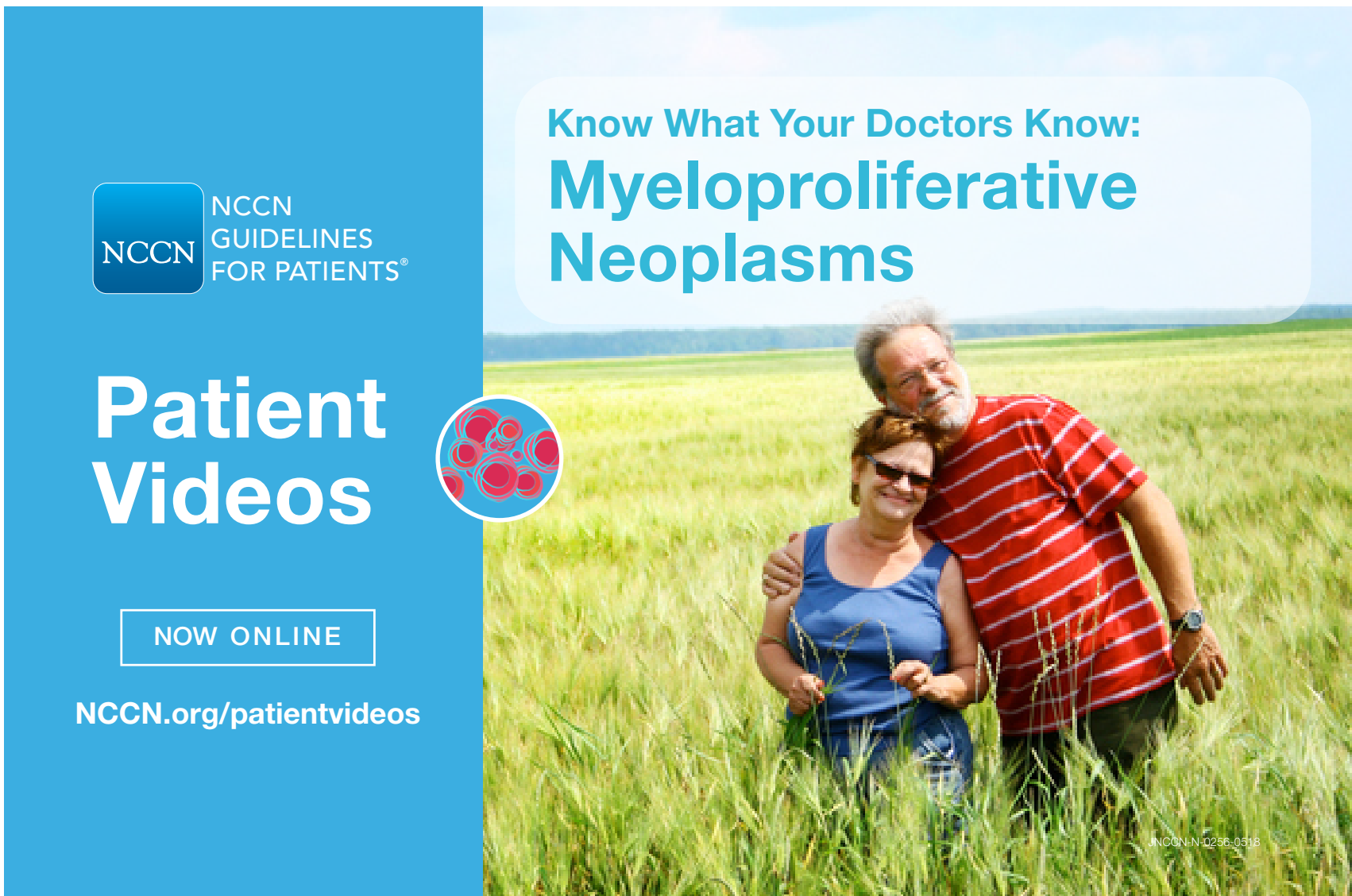

